\title{
El poblamiento de Baja California y la influencia de la política de población en el periodo cardenista
}

\author{
Norma del Carmen Cruz González*
}

\begin{abstract}
Resumen: Baja California creció de manera vertiginosa durante el siglo XX con un promedio anual de 5.8\%, el más alto a nivel nacional. Para explicar el proceso de su poblamiento, existe la idea de que las políticas centrales no influyeron de manera directa, sino que éste se debió a procesos gestados desde mediados del siglo XIX, así como a las relaciones de dependencia e intercambio desigual con California, Estados Unidos. Sin embargo, aquí se analiza la implementación de la política de población por parte del Estado mexicano, particularmente en el régimen del presidente Lázaro Cárdenas, quien propugnó una "colonización" con mexicanos en Baja California. Se examina igualmente el impacto de dicha política en el crecimiento de la población.

Palabras clave: Baja California, política de población, colonización, cardenismo, poblamiento
\end{abstract}

Abstract: Baja California's demographic growth rate during the $20^{\text {th }}$ century was vertiginous: at an annual average of $5.8 \%$, it was the highest rate in the country. This phenomenon has been generally explained as a result of processes beginning in the mid $19^{\text {th }}$ century as well as of dependency relations and the trade imbalance with California. The direct influence of central policies has been, to a great extent, ignored. Therefore, this article analyzes the way in which the Mexican state implemented population policies and their impact. In particular, it focuses on the Lázaro Cárdenas regime, when the "colonization" of Baja California with Mexicans was strongly advocated.

Key words: Baja California, population policy, colonization, cardenism

* Investigadora del Instituto de Investigaciones Históricas de la Universidad Autónoma de Baja California. Correos electrónicos: tascari_11@yahoo.com, nortwo@gmail.com 


\section{Introducción}

El objetivo de este artículo es analizar la influencia de la política de población del periodo cardenista y específicamente de la Ley General de Población de 1936 en el crecimiento de Baja California. La utilización de un método cuantitativo y de un periodo que trasciende la década de 1930 y 1940, nos permitió estudiar desde una perspectiva de mayor duración el crecimiento de la población y los hechos que se relacionan con tal política, implementada y desarrollada subsecuentemente en las décadas posteriores.

El cardenismo ha sido considerado un periodo de atención a demandas agrarias y parte del proceso de estabilización de instituciones sociales y políticas que tuvo inicio desde el gobierno de Plutarco Elías Calles (Anguiano, 1984). En este periodo se normaron los aspectos poblacionales al expedirse la Ley General de Población en 1936. Esto significa que la problemática poblacional en su conjunto adquirió relevancia a nivel nacional y se regulaba a partir de la creación de esta ley. Este hecho encaminó los esfuerzos de la política hacia el objetivo de poblar el país, por lo que se fomentó el crecimiento natural y la distribución racional de la población.

Un ejemplo de dicha política fue Baja California, que vio acrecentar su población de manera veloz durante el siglo xx. Un promedio general anual de $5.8 \%$ en la tasa de crecimiento durante este siglo, a diferencia del total para el país que tuvo una tasa del $2 \%$, coloca a la entidad en la cima del crecimiento poblacional en todo México. Dentro de las características del poblamiento bajacaliforniano está el incremento poblacional a partir de la migración, ya que, sin excepción, sus tasas netas migratorias fueron positivas. Otras particularidades son que dicha población se desarrolló en un entorno urbano que se encontraba paralelo a la zona de la frontera con Estados Unidos.

Si atendemos a que el reparto agrario y la política de poblamiento fueron dos objetivos del cardenismo, es válido preguntarse si dichas líneas de acción influyeron en el poblamiento de Baja California. Hay que resaltar que tanto el carácter fronterizo de la entidad en virtud de su vecindad con California, Estados Unidos, como su distancia respecto al centro político del país, son elementos que han sido tomados 
en cuenta para argumentar dos posturas. La primera es abordada por Alejandro Canales (1995: 20-21), quien afirma que existe una relación de dependencia económica de Baja California hacia California que obstaculizó la influencia de las políticas del gobierno central en el crecimiento de la población y el desarrollo económico de la región. La otra postura es de Marco Antonio Samaniego (1999a: 639), quien dice que el reparto agrario sí tuvo una influencia significativa en la colonización bajacaliforniana. Nosotros estamos de acuerdo con esta última perspectiva, pero consideramos que no es suficiente un análisis con elementos cualitativos, sino que es necesario complementarlo con un análisis cuantitativo del crecimiento de la población y la importancia de la instrumentación de políticas demográficas a partir de la Ley General de Población de 1936.

\section{Contexto demográfico histórico de Baja California}

La península de Baja California fue objeto de proyectos colonizadores durante el siglo XIX (Piñera, 1991). En el Porfiriato se favorecieron las inversiones de compañías extranjeras para la explotación de recursos agrícolas, ganaderos o mineros. En cuanto a la población, en 1887 se sumaron 34668 habitantes: 21738 en el Partido Sur, 5891 en el Partido del Centro, y 7039 en el Partido Norte. ${ }^{1}$ Una de las características de este periodo es que el sur de la península estaba más poblado que el norte. El crecimiento poblacional de la parte norte se vio favorecido por la prosperidad económica del sur del estado de California, Estados Unidos, así como por el decaimiento de la minería en la parte sur que llevó a muchos a emigrar hacia esta zona; asimismo los inicios de la Revolución en el centro del país influyeron en el movimiento de la población hacia la frontera norte (Lemoine, 1959: 265-267). Tijuana y Mexicali son ejemplos de este proceso, ya que ambas localidades crecieron de manera vertiginosa en la primera mitad del siglo xx.

\footnotetext{
${ }^{1}$ Por decreto del 14 de diciembre de 1887 se volvió a dividir al Territorio de la Baja California en dos Distritos Políticos, el Sur y el Norte. El primero integró a los Partidos del Centro y del Sur y el segundo al Partido Norte (INEGI, 1997: 52-53).
} 
Durante la primera mitad de ese siglo existió un crecimiento natural alto y un constante flujo migratorio en Baja California. Como se observa en la figura 1, en este periodo existieron dos décadas en las que aumentó con mayor énfasis la población, mismas que se han explicado de manera parcial en la historiografía bajacaliforniana: la que va de 1910 a 1920, con una tasa de $7.9 \%$, y la de 1940 a 1950, con una del $10.3 \%$. A partir de esta última década la población en Baja California experimentó un descenso -aunque con tasas altas- mientras que la población nacional aumentaba. Esto sucedió hasta la década de 19701980, en la que los porcentajes reportaron mínimas variaciones que de acuerdo a la gráfica parecieran unirse en el mismo punto, de 3.1 y $2.9 \%$ del país y la entidad respectivamente; a partir de ahí las tasas se disocian: la de Baja California asciende y la nacional entra en descenso (figura 1).

Entre los hechos que explican el crecimiento de Baja California en la década de 1910 a 1921, de manera general se encuentran el auge de

Figura 1.

Tasas de crecimiento de la República Mexicana y de Baja California, 1895-2000

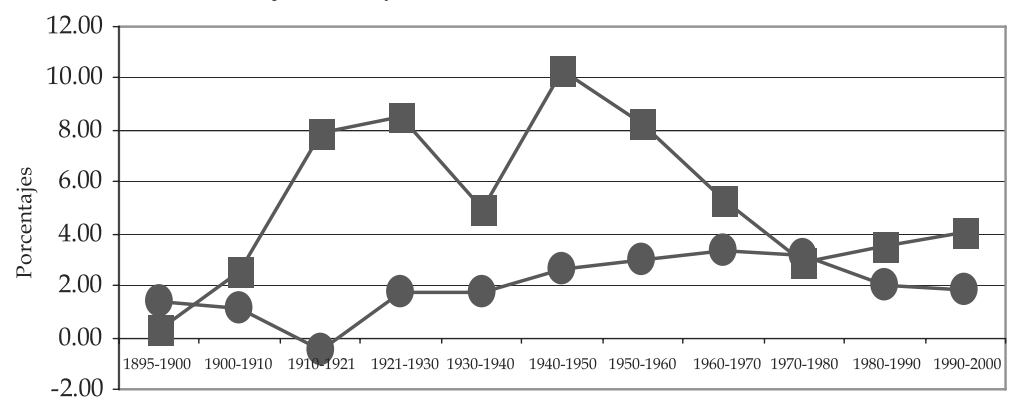

Años intercensales

$\multimap$ - República Mexicana

- Baja California

Fuente: Cálculos propios a partir de los Censos de Población y Vivienda, 18952000. Las cifras corresponden a las siguientes fechas censales: 20 de octubre (1895); 28 de octubre (1900); 27 de octubre (1910); 30 de noviembre (1921); 15 de mayo (1930); 6 de marzo (1940); 6 de junio (1950); 8 de junio (1960); 28 de enero (1970); 4 de junio (1980); 12 de marzo (1990); 5 de noviembre (1995); y 14 de febrero (2000). 
la producción algodonera en Mexicali y el fomento de la inmigración china para el trabajo agrícola; en menor medida, se debió también a que la entidad recibió a los que venían huyendo de la Revolución. En cuanto a las razones que provocaron el aumento poblacional entre 1940 a 1950 está la apertura del programa Bracero y la construcción e inauguración del ferrocarril Sonora-Baja California.

La década de 1930 a 1940 tuvo una tasa de crecimiento de 5\%, misma que se encuentra en medio de las tasas más altas ya mencionadas. Lo anterior nos lleva a preguntarnos sobre la forma en que se desarrolló el crecimiento bajacaliforniano durante la primera mitad del siglo, debido a que de un comportamiento de alta inmigración entre 1921 y 1930, se pasó a uno de más baja intensidad entre 1930 y 1940, para volver a dispararse en la siguiente década. En esta última década de 1930 a 1940 se implementó la Reforma Agraria y con ella una redistribución poblacional. Sin embargo, en Baja California se experimentó un aparente descenso en contraste con las dos décadas anteriores. El comportamiento esperado, en términos demográficos, sería una congruencia en el aumento entre 1921 y 1950, aun cuando hubo eventos que influyeron en que creciera menos de lo esperado, si bien siguió en ascenso. Entre los acontecimientos que pudieron influir en dicho crecimiento está la depresión económica de 1929, la derogación de la Ley Seca en Estados Unidos en 1933 y las restricciones en el ingreso de extranjeros al país, sobre todo de asiáticos, así como su salida de la entidad. ${ }^{2}$

\section{Baja California, entidad de pocas pero heterogéneas municipalidades}

Baja California es una entidad de exiguas pero heterogéneas municipalidades. ${ }^{3}$ Los poblados considerados más importantes son los que hasta este momento constituyen los municipios y ciudades con la

\footnotetext{
${ }^{2}$ Entre 1922 a 1924 se intensificó la propaganda antichina a través de comités Proraza en Sonora; diez años después, la campaña antichina se llevó a cabo en Baja California (Velázquez, 2001: 240 y 286).

${ }^{3}$ Se determinó usar la categoría localidad como término que puede ser utilizado tanto para designar a una ciudad como a un pueblo, debido a que para una des-
} 
mayor cantidad de población: Ensenada, Mexicali, Tecate y Tijuana. ${ }^{4}$ En el cuadro 1, por tanto, se engloba la información municipal y por localidad de estos cuatro principales centros de población de la entidad durante el siglo xx. El municipio de Ensenada, fundado en 1882, fue la tercera cabecera política, después de Santo Tomás de la Fronte$\mathrm{ra}^{5}$ y de Real del Castillo. Ésta mantuvo en 1900 una población de 4327 habitantes. Para este momento, sólo por localidad Ensenada registró a 1726 habitantes, mientras que en Tecate y Tijuana la cantidad era mínima, de 127 y de 242 respectivamente. Si bien sólo se incluyó la información de las cuatro principales localidades en el cuadro, existieron para principios del siglo siete secciones municipales: Ensenada, Tijuana, Mexicali, El Álamo, Santo Tomás, El Rosario y Calmallí; además de las comisarías: Tecate, Los Algodones, Real del Castillo, San Telmo y San Quintín. Las secciones municipales apenas alcanzaban los 200 habitantes, lo cual era un requisito de esta categoría (González Félix, 2002: 13).

Para inicios del siglo xx, en Ensenada tanto la población como la economía habían decrecido, lo que se notó en el estancamiento poblacional registrado en el periodo censal 1895-1900, pasando su población en cuanto a nivel localidad de 2113 en 1895 a 1726 habitantes en $1900 .{ }^{6}$ Algo similar sucedió de 1910 a 1921, donde se registró el aumento de ocho personas en el periodo intercensal, de 2170 a 2178 habitantes. $^{7}$

cripción del proceso del crecimiento local de Baja California, el término municipio se torna inadecuado ya que propicia la pérdida de información de algunos de los lugares más importantes. Por tal motivo se realizó una serie histórica de población con las dos modalidades, tanto por municipio como por localidad.

${ }^{4}$ Playas de Rosarito formó parte de Tijuana hasta 1995, cuando se convirtió en municipalidad. En el cuadro 1 sólo se incluye en el total de la entidad en el Censo del 2000.

${ }^{5}$ Municipalidad por decreto del 2 de diciembre de 1855 y derogada el 24 de diciembre de 1873 (INEGI, 1997: 73).

${ }^{6}$ La información de la población de Ensenada de 1895 fue tomada de Rosela Bonifaz (1999: 343) y no se encuentra en el cuadro 1.

${ }^{7}$ A finales del siglo XIX la minería entró en decadencia en Ensenada, constituyendo una de las razones de la reducción de su población. Para el siglo XX, entre las razones a que se atribuye este decaimiento fue el cambio de cabecera política, que desde 1915 había realizado el coronel Esteban Cantú al ingresar como jefe político del Distrito, lo cual conllevó un atraso económico en Ensenada. Otra razón fue la 


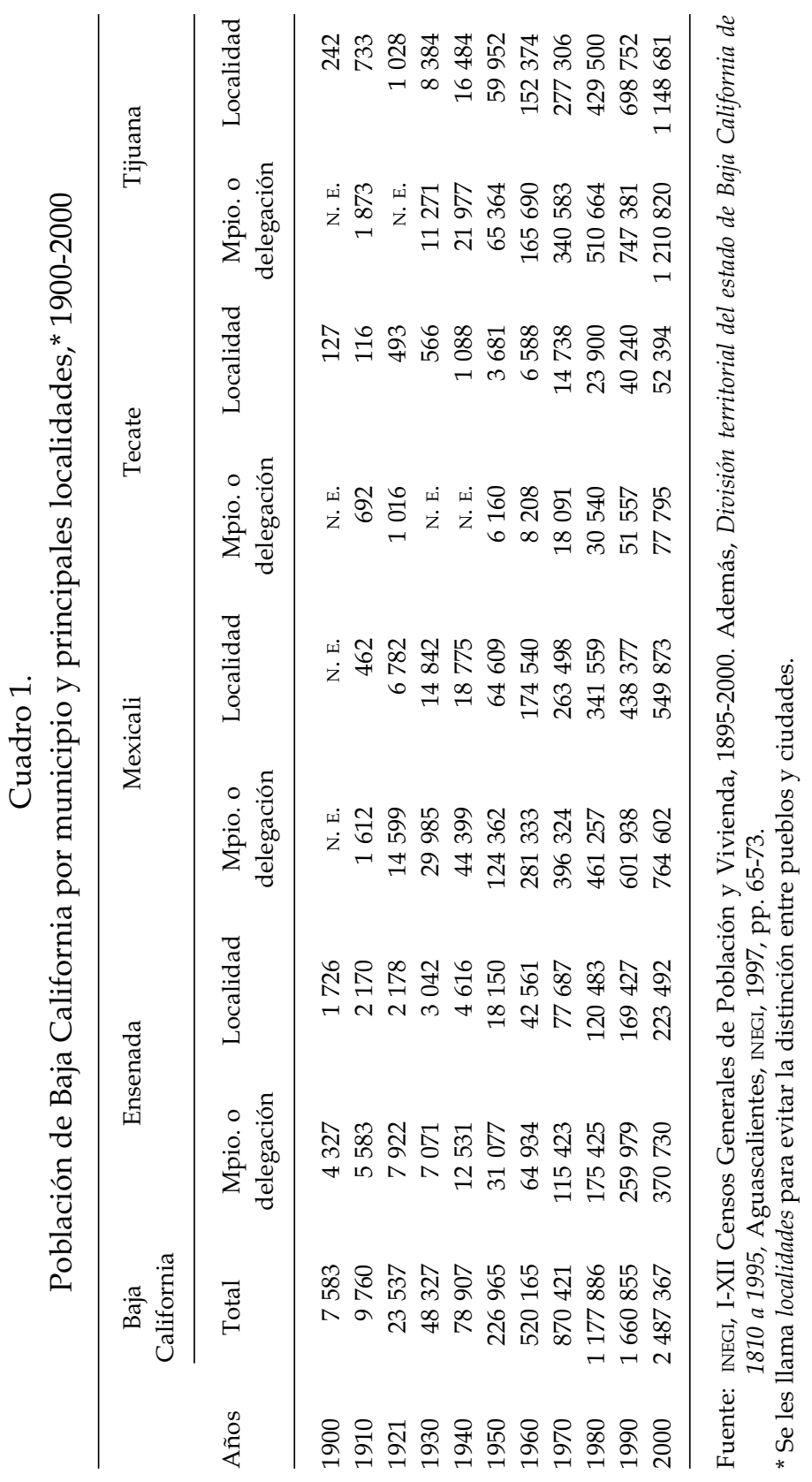


Mexicali y Tijuana, por su parte, eran aldeas o vecindades con alrededor de 177 y 300 habitantes en 1904 y 1905 respectivamente (Walter Meade, citado en González Félix, 2002: 15). Tijuana nació en 1889 a raíz de las visitas de extranjeros a las aguas termales que había en el poblado, lo que la ubicó como centro turístico. Mexicali, por su parte, reportó sus primeros habitantes después de 1901, en un "conjunto de viviendas improvisadas y ramadas". Después llegaron las medidas prohibicionistas de moralistas y puritanos a California, las que influyeron para que se crearan en la zona fronteriza, es decir, en Mexicali y Tijuana, una serie de comercios de venta de licores y de servicios como el juego y la prostitución (González Félix, 2002: 13-16). No obstante la cantidad de habitantes, Tijuana y Mexicali fueron catalogadas como ciudades desde $1989 .{ }^{8}$

Para observar el incremento de la población, en la figura 2 se aprecian las tasas de crecimiento anual de las localidades principales de Baja California. Durante el curso del siglo pasado el crecimiento fue acelerado: como ciudad al final del siglo (1990-2000), Tijuana mantuvo una tasa de crecimiento del $5 \%$, mientras que su promedio anual ascendió al 8.8\% durante los cien años. Lo anterior se debe a que la tasa más alta registrada la obtuvo en el decenio que va de 1921 a 1930 de $24.8 \%$, seguida del $6.9 \%$ en el siguiente decenio de 1930-1940, para ascender nuevamente a una tasa de $12.6 \%$ entre 1940 y 1950.

También resalta Mexicali con un promedio anual en el siglo de 7.7\% como localidad, registrando su más alto crecimiento en la década 1910-

devaluación del peso al dos por uno respecto al dólar, lo que aumentó el costo del combustible que se consumía en la entidad ya que era pagado en oro (Bonifaz de Novelo, 1999: 471-489). Por otro lado, no se puede descartar la existencia de errores de calidad en los datos estadísticos históricos, sobre todo en lo referente a la suma de sólo ocho habitantes de 1910 a 1921 en Ensenada, aunque se trabajaron sin ajustarse por ser fuente primaria de información.

${ }^{8}$ Según el INEGI (1997), se consideró a Tijuana y Mexicali como "ciudades" a partir de 1989, sin embargo, la cantidad de 2500 habitantes la alcanzó Mexicali hasta 1921, y Tijuana hasta 1930. En el caso de Tecate, tuvo diversos cambios de adscripción en este tiempo. Primero se constituyó como municipio en 1917, seis años más tarde, en 1923, fue anexado al de Mexicali. En 1925 perteneció a Tijuana. Para 1947 se instituyó como delegación y, finalmente, en 1953 Tecate se convirtió en municipio y fue considerado una ciudad a partir de ese año. 
Figura 2.

Tasas de crecimiento de las principales localidades de Baja California, 1900-2000

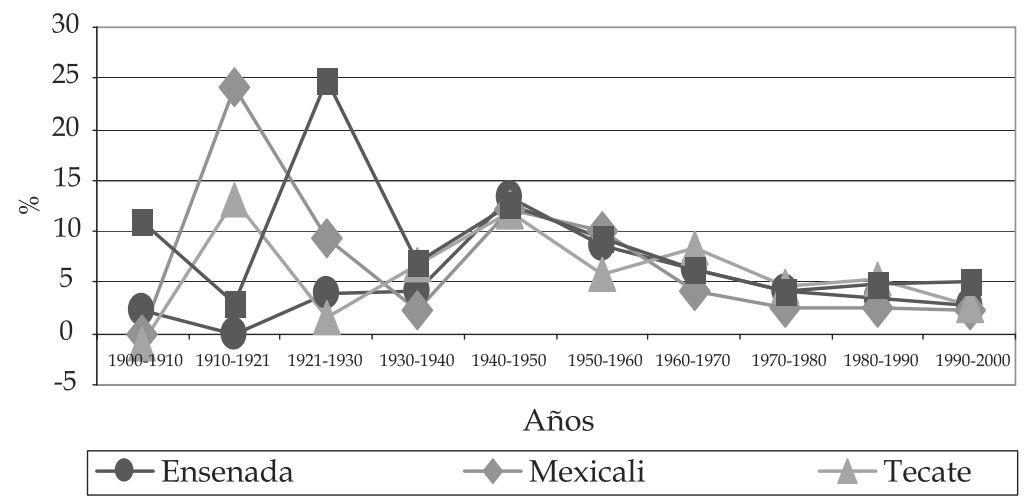

Fuente: Cálculos propios a partir de los Censos de Población y Vivienda, 19002000.

1921 con una tasa de $24.2 \%$, esto es, 10 años antes que Tijuana. En el decenio de 1940-1950 vuelve a sobresalir con una tasa de $12.1 \%$; mientras que en el correspondiente a 1921-1930 ascendió a 9.3\% y en el de 1930-1940 sólo alcanzó el 2.4 por ciento.

Las únicas localidades que registraron un descenso en el periodo de la lucha armada entre 1910 y 1921 fueron Ensenada y Tijuana. Ello contrasta con el comportamiento de Mexicali en este periodo, que fue el ascenso de la tasa a $24.2 \%$, como ya se mencionó. Por su parte, Tecate creció en $13 \%$. Sin embargo, no debe olvidarse que se han estado analizando los datos de las localidades, no de las municipalidades.

Ensenada, por su parte, registró en este periodo un crecimiento moderado de $3.2 \%$, a diferencia del $20 \%$ de Mexicali. Por lo tanto, esta última municipalidad llevó la delantera del crecimiento poblacional en el periodo 1910-1921 al sumar 1612 habitantes, cifra que ascendió en ese decenio a 14599 habitantes. Mexicali contó con compañías que sembraban y exportaban el algodón, entre ellas la Colorado River Land Company, que se convirtió en una de las principales empresas que 
explotaron el Valle de Mexicali desde 1902. Para 1912, el paulatino crecimiento económico generó la demanda de mano de obra agrícola, para lo cual se inmigró a trabajadores chinos. Esto significa que para 1920 se alcanzó a inmigrar hasta 4 mil chinos y 5889 en 1928 (Velázquez, 2001: 73 y 279).

La inestabilidad del país, característica del periodo revolucionario, fue subsanada a nivel regional a partir del fortalecimiento de la hacienda pública mediante la explotación del algodón, el comercio de licores y bebidas embriagantes y los ingresos derivados a propósito de la inmigración china, además de la apropiación de terrenos por parte del gobierno (González Félix, 2002: 56). La recaudación de dinero permitió la edificación de escuelas, puentes y oficinas públicas en Mexicali, así como la elaboración de la traza de la carretera de Mexicali a Tecate y Tijuana (Bonifaz de Novelo, 1999: 490). Pero existía la disputa política entre el gobernador del Distrito y las autoridades de Mexicali y Ensenada por los ingresos que se generaban en Tijuana a raíz de los casinos, el hipódromo y los expendios de bebidas alcohólicas, ya que esta última no era municipio sino que se encontraba en calidad de delegación municipal (Samaniego, 1999d: 517). Este crecimiento económico en Tijuana se debió a que en 1917 el movimiento prohibicionista o temperante adquirió relevancia a nivel nacional en Estados Unidos, y por consiguiente entró en vigor la Ley Volstead o Ley Seca en 1920, misma que fue derogada hasta 1933, lo que produjo en esos años el incremento del sector terciario y el aumento de ingresos en esta localidad (González Félix, 2002: 14 y 17).

Después del periodo de gobiernos civiles en la entidad, ${ }^{9}$ el cual duró de 1920 a 1923, Abelardo L. Rodríguez ingresó como gobernador al Distrito Norte, en el que permaneció hasta su renuncia en 1929. Para justificar el dinamismo del desarrollo capitalista en Baja California, Rodríguez afirmó que el "Estado no combatía a la burguesía, la fomentaba, la apadrinaba y la convertía en una posibilidad de cre-

\footnotetext{
${ }^{9}$ Por "gobiernos civiles" se conoce el periodo que va de agosto de 1920 a noviembre de 1923, en el cual tomaron posesión gobernadores que no eran militares. Éstos fueron: Luis M. Salazar, Manuel Balarezo, Epigmenio Ibarra Jr., Lucas B. Rodríguez y José Inocente Lugo (Samaniego, 1998; Calvillo, 1994).
} 
cimiento para la economía" (Samaniego, 1999c: 593), ${ }^{10}$ por lo que el empresariado regional se vio acrecentado y fortalecido en ese momento. Por otro lado, a pesar de fomentar la injerencia de estadounidenses en la economía regional, el general Rodríguez enarboló como objetivo la "nacionalización" de la frontera México-Estados Unidos, considerada como una "misión patriótica" a la cual se refería con la denominada "mexicanización" de Baja California. Asimismo, el general Rodríguez mencionaba que su propósito era "fomentar el arraigo y progreso de los agricultores mexicanos y redimir las mayores extensiones de terreno por medio de compras efectuadas a los latifundistas extranjeros, de acuerdo con las posibilidades del tesoro local". Otra de las razones fue la existencia de un "marcado ambiente de extranjerismo", debido a que las compañías extranjeras poseían la mayor parte de las tierras irrigables. Durante su administración se ejecutaron, entre otras cosas, dos medidas en cuanto al problema de la colonización en el Distrito: la primera fue el fomento de la repatriación de elementos nacionales, y la segunda la creación de cooperativas para el desarrollo económico de la región. Así lograría o procuraría su "engrandecimiento" (Rodríguez, 1993: 122-123).

Como ya se mencionó, Tijuana alcanzó su más alto índice poblacional con la tasa de $24.8 \%$ en la década de 1921 a 1930, mientras las tasas de Mexicali y Tecate descendieron y la de Ensenada aumentó de 0.03 a $4 \%$. Aquí se observa un cambio más real de la población ensenadense, ya que de haber sumado sólo ocho personas de 1910 a 1921, al menos en las cifras oficiales, se pasó a 3042 habitantes en 1930.

Para el decenio de 1930 a 1940, a pesar de aumentar casi al doble su población de 8384 a 16484 habitantes, Tijuana tuvo una tasa de crecimiento de $6.9 \%$, en comparación con el $24.8 \%$ de la década anterior. En el mismo periodo, la tasa de Mexicali siguió en descenso al pasar del 9.3 a $2.4 \%$; es decir, el aumento no fue tan significativo ya que su población pasó de 14842 a 18775 habitantes. Por su parte, Tecate y

\footnotetext{
${ }^{10}$ Un trabajo más amplio acerca del periodo de Abelardo L. Rodríguez y el estado en que se encontraba el Distrito Norte de la Baja California se encuentra en Gómez (2002).
} 
Ensenada aumentaron su población a nivel localidad en este periodo. La tasa de crecimiento de Tecate subió de 1.6 a $6.7 \%$, aunque la de Ensenada sólo aumentó tres décimas, de 4 a 4.3\%. Por tanto, Mexicali y Tijuana marcaron la pauta para que la tasa de crecimiento global de la entidad disminuyera al 5\%, después del $8.5 \%$ registrado en el periodo intercensal anterior.

El contexto de los años treinta en Baja California se caracterizó por la llegada de la crisis económica, así como por la idea de que Estados Unidos quería apoderarse de la península, la cual se mantenía vigente en el pensamiento de los pobladores desde mediados del siglo XIX. Asimismo, un marcado extranjerismo en las poblaciones fronterizas influyó para que se implementara un "Plan de mexicanización" que buscaba promover la colonización con trabajadores mexicanos; reducir y prohibir la inmigración china; que se efectuaran las transacciones comerciales con moneda mexicana, que se cambiaran los anuncios al idioma español; y que se recuperaran las tierras en posesión de la Colorado River Land Company (Samaniego, 1999b: 597-599).

En la década de 1940 a 1950, todas las localidades se encontraron dentro de un mismo rango de crecimiento. Tecate obtuvo una tasa de $11.9 \%$, Ensenada de $13.4 \%$, Tijuana de $12.6 \%$ y Mexicali de $12.1 \%$. Ésta es la última cúspide poblacional registrada en la que todas las localidades mantuvieron un crecimiento similar y sostenido para emprender el descenso después del boom poblacional. Mientras tanto, Baja California superó por una diferencia de +148058 habitantes de 1940 a 1950, lo que correspondía al aumento del $288 \%$ de su población al pasar de 78907 a 226967 habitantes. Éste fue el preámbulo que necesitaba para formar parte de la lista de los estados soberanos de la República, y con ello fortalecer a los sectores industrial y de servicios.

Uno de los requisitos para la conversión de territorio a estado era alcanzar la cantidad de 80 mil habitantes, según la Constitución de 1917, lo cual Baja California logró hasta 1950 (Taylor, 2002: 165), como ya se mencionó. Para el Estado cardenista, durante cuya administración no se había alcanzado ese número de población, era preciso que los territorios de Baja California Sur y Norte, y Quintana Roo, fortalecieran su poblamiento, quedaran "integrados" al país y salvaguardaran las fronteras territoriales. La primera Ley de Población y el discurso 
de Lázaro Cárdenas sobre los territorios ${ }^{11}$ dieron por sentada dicha consideración.

\section{Elementos de las políticas de población}

Para el análisis de la política de población en Baja California se retoma la definición de Gilberto Martínez (1987), quien afirma que se trata de la implementación de medidas tomadas por el Estado como una respuesta que obtiene consenso social y voluntad política a partir de la organización de las instituciones y la legislación a los problemas demográficos. Se reitera este concepto para el análisis de la forma en que se llevó a cabo el poblamiento de Baja California, ya sea que haya influido la política de población o bien que haya sido producto de la inercia demográfica.

Las políticas de población son leyes o reglamentos que tienen como propósito modificar las conductas demográficas. Para el periodo que aquí se describe, la existencia de un interés explícito por parte del Ejecutivo nacional no era suficiente en la medida en que no estaban resueltas otras cuestiones como la distribución de las tierras y las vías de comunicaciones para facilitar el acceso a la península por vía terrestre. Por tanto no bastaba la intención de colonizar la frontera bajacaliforniana, sino que habrían de crearse las condiciones que permitieran el acceso y el arraigo necesario para que perduraran y crecieran las poblaciones fronterizas. En este sentido, la nacionalización y el reparto de tierras a través de la Reforma Agraria fue una solución para poblar con "elementos nacionales" a la entidad. Es por eso que desde un principio se formó una Comisión Intersecretarial12 ${ }^{12}$ con el objetivo de establecer los requerimientos específicos para la entidad e

\footnotetext{
11 "Exposición del presidente de la República sobre la reconstrucción integral de los territorios de Baja California y Quintana Roo. México, D. F., 28 de septiembre de 1936" (Cárdenas, 1978a: 215-222). En adelante nos referiremos a este documento como Plan de Recuperación (1936).

${ }^{12}$ Este organismo fue creado por Lázaro Cárdenas para analizar la situación bajacaliforniana y fue compuesto por personas de todas las secretarías de Estado: de Hacienda, Salubridad, Economía, Agricultura y Fomento, Comunicaciones y Transportes y un representante del gobernador del Territorio. Si bien Samaniego menciona que se creó en octubre de 1935, desde 1930 se constituyó una comisión
} 
informar la presidencia de lo que acontecía y era pertinente realizar en Baja California.

Es decir, para que se considere que una política de población se llevó a cabo -en este caso en Baja California- debe haber una correspondencia entre la definición en abstracto de dicha política y los hechos. En este sentido, Carmen Miró (1975) enumeró algunos puntos medulares como requisitos que toda política de población debe tener. Asimismo, éstos también son prioridad en las metas de desarrollo social y económico de un país, tal como lo afirma esta autora. Los puntos principales que enuncia son los siguientes: 1 . que sea una decisión del sector público; 2 . un sujeto al que va dirigida; 3 . un reconocimiento del curso que se desea sigan los acontecimientos (o la conducta con el propósito de lograr el objetivo deseado); 4. una línea de acción; 5. una declaración de intención o qué se pretende alcanzar, cómo y por qué y; 6. la instrumentación (1975: 4). La intención de colonizar Baja California cumple esos requisitos de una política de población. De este modo, adoptando la propuesta de Miró para el análisis del proceso de poblamiento bajacaliforniano que impulsó el régimen cardenista, podemos realizar las afirmaciones siguientes.

\section{1) Decisión del sector público}

Baja California vivía un momento de incertidumbre política durante la década de los años treinta. En ésta desfilaron diez gobernadores del Distrito y posterior Territorio de la Federación. Sólo en el año de 1930 existieron tres administraciones y en la última de este año, a cargo del licenciado Carlos Trejo y Lerdo de Tejada, se realizó el cambio de categoría política de la entidad, en la cual Baja California dejó de ser Distrito para convertirse en el Territorio Norte. ${ }^{13}$

antecesora que tenía el mismo objetivo de conocer las condiciones generales que aquejaban a Baja California (AGN, Dirección General de Gobierno, serie 2.300(30)10, caja 157, exp. 18, en IIH-UABC, [16.35]). Además, existieron algunos informes acerca de las condiciones de Baja California que se realizaron en estos años; en 1933, por ejemplo, Julio Riquelme publicó El Territorio Norte de la Baja California y sus principales problemas (SMGE, IIH-UABC, [2.31]).

${ }^{13}$ Por decreto del 7 de febrero de 1931, se declaró que en lo sucesivo el paralelo $28^{\circ}$ de latitud Norte sería la línea divisoria entre el Territorio Norte y el Territorio Sur 
Lo anterior, como ya se dijo, fue un paso más dentro del área administrativa, puesto que no promovió cambios sustanciales o diferencias significativas y se seguían compartiendo las mismas leyes y reglamentos con el Distrito Federal. En ese sentido, se infiere que aunque Baja California no podía constituirse todavía como estado por falta de población, se cambió su categoría política a pesar de que siguiera bajo el mando directo del Ejecutivo nacional y de que los problemas que aquejaban al Distrito Federal no fueran comparables a los de los territorios. $^{14}$

Así que en calidad de territorio dependiente del Ejecutivo Federal, Baja California y Quintana Roo fueron objeto de un manifiesto expresado por el presidente Lázaro Cárdenas en 1936, al cual se le ha denominado "Plan de recuperación de los territorios" (Cárdenas, 1978a). A grandes rasgos, en dicho documento se advierte que constituían un problema en tanto permanecían aislados debido a su situación geográfica y expuestos a las ambiciones que despertaban sus recursos naturales. Expresaba también que solamente 106030 habitantes vivían en 194356 km², abarcando Quintana Roo y Baja California, lo cual no permitía un intercambio mercantil ni cultural, ni para hacer producir la riqueza.

Cárdenas planteaba realizar un programa constructor que, por medio de poblar estos territorios, contribuyera a su integración a la nación.

de la Baja California (INEGI, 1997: 43). Carlos Trejo y Lerdo de Tejada afirmó que el suceso del cambio de categoría no tuvo mayor trascendencia que la de constituir un acto legislativo (Samaniego, 1999b: 610). Sin embargo, Max Calvillo (2002) afirma que desde la expedición de la Ley de Organización del Distrito y Territorio Federales en 1917 se tomaba a la península como un solo territorio y no se hacía mención de la división de los dos partidos; con lo cual inferimos que existía un desconocimiento de Baja California por parte del centro del país y que dicha transformación sirvió para reafirmar la división de los territorios (INEGI, 1997: 61-62).

${ }^{14}$ Enrique P. Gessenius y Tomás Ojeda, presidente y secretario de la Asociación Cívica Pro-Baja California, enviaron al Ejecutivo un informe sobre las condiciones que prevalecían en Baja California. En dicho informe afirmaban que era un "error grave que se ha cometido, al hacer extensivos para que rijan en los Territorios de la Baja California y de Quintana Roo, las leyes y reglamentos que están en vigor en el Distrito Federal; teniendo como tienen, condiciones y problemas tan diferentes [...]" (16 de abril de 1936, AGN, fondo Lázaro Cárdenas, vol. 488, exp. 437.1/413). 
Dicha integración, en palabras del presidente, promovería que tanto Quintana Roo como Baja California

[...] vivan con el ritmo económico y social de nuestra nacionalidad y mantengan y afirmen las características de la cultura patria, en lugar de seguir luchando desventajosamente, como lo hacen, por neutralizar el efecto de los contrastes que se establecen en los lugares inmediatos a comarcas extranjeras de estructura económica más evolucionada (Plan de Recuperación, 1936, en Cárdenas, 1978a: 215).

\section{2) Sujeto al que va dirigida}

A diferencia de la colonización promovida por el Porfiriato y por los gobiernos conservadores en la segunda mitad del siglo XIX, la política de colonización cardenista iba dirigida a poblar los territorios con "elementos nacionales". Ello se puede evidenciar a través de lo expuesto en la Ley General de Población para el país, pero también en el Plan de Recuperación del presidente al mencionar que "el esfuerzo del Ejecutivo $[. .$.$] tendrá por objeto poblar en la medida necesaria, con ele-$ mentos nacionales, los territorios sur y norte de la Baja California y el de Quintana Roo [...]" (Cárdenas, 1978a: 215).

Por otro lado, dentro del artículo $6^{\circ}$ de la Ley General de Población se estipula que a los repatriados se les debía ubicar donde fueran de mayor utilidad, dependiendo de las capacidades adquiridas en el país donde hubieran vivido. En el caso de Baja California, se impulsó el estudio de las condiciones de la entidad para recibir a repatriados y ubicarlos dentro de colonias agrícolas y en el trabajo de construcción de la Presa Rodríguez. No obstante, el número de repatriados que recibieron tierras en Baja California fue reducido, según Alanís. ${ }^{15}$

\footnotetext{
${ }^{15}$ Si bien Fernando Alanís (2000) menciona que no hubo grandes dotaciones de tierras a los repatriados, como se especuló en los diversos estudios, en uno de ellos se cita al secretario de Agricultura Saturnino Cedillo, quien en 1935 habló de la posibilidad de repatriar alrededor de 10 mil familias de Los Ángeles, California, aunque primero debían resolverse los problemas de la falta de agua y regadío. Alanís señala que se les otorgaron facilidades a pequeños grupos de repatriados en calidad de colonos en Mexicali, que su número fue reducido y las tierras otorgadas también, pero no da a conocer el número de éstos (pp. 145-159), quizá porque no se han encontrado o no existen tales registros.
} 


\section{3) Conducta dirigida con el propósito de lograr el objetivo deseado}

Uno de sus propósitos fue redistribuir la población altamente concentrada en el centro del país hacia territorios menos poblados, y en el caso de Baja California, promover el arraigo de sus habitantes con objeto de salvaguardar la soberanía nacional e impedir una nueva invasión por parte de Estados Unidos.

La escasez de población fue vista como un problema de integración nacional, tal como lo manifestó Gilberto Loyo (1935) en su Política demográfica, quien marcó como un hito el año de 1933. Las razones que pudieran explicar este hecho se deben en primera instancia a que un año antes fue creado el Comité Mexicano para el Estudio de los Problemas de la Población, además de que Loyo publicó algunos de sus artículos en el periódico El Nacional en 1932. Por otro lado, Loyo hizo hincapié en el discurso pronunciado por el general Calles acerca de la incorporación del problema demográfico a la ideología revolucionaria en calidad de fundamental, curiosamente, después de su regreso de Baja California. Esto implicó que el problema demográfico se inscribiera en el Plan Sexenal del PNR. Además, pronunció que la distribución de la población se pretendía lograr a través de una política de distribución de la población rural y de colonización interior con nacionales, asimismo, la proposición de mejorar las condiciones sanitarias se veía mermada ante la baja densidad demográfica, la cual se convertía en "un serio obstáculo para el desenvolvimiento del país" (Loyo, 1935: 23-25).

El hecho de que Calles se hubiera encontrado en Baja California antes de pronunciar ese discurso señala una especie de precedente de que el gobierno central, en este caso Calles como jefe máximo y Rodríguez como presidente y ex gobernador de la entidad, reconocía a nivel nacional "el problema demográfico". A este poblamiento de regiones de baja densidad poblacional había que hallarle una solución que, además, prometiera un desarrollo económico.

Si bien desde 1911 se temía en Baja California una invasión estadounidense, para la década de los treinta existían algunas dificultades entre las autoridades que querían implantar medidas del centro del país y los empresarios de la región. La crisis económica tuvo efectos 
en la economía regional, por lo que se solicitó la exención de impuestos fiscales en la entrada de productos extranjeros al país, lo que beneficiaba a los comerciantes pero no al campesinado, que dejó de cultivar algunos productos debido a la competencia que representó el mercado estadounidense. Los repatriados se convirtieron en un problema de sobrepoblación al momento de la crisis y la prohibición de los juegos de azar, y los chinos eran vistos como competencia para los mexicanos en el comercio local (Samaniego, 1999b: 597-638).

Lo anterior fue el preámbulo a la política cardenista, que inició en 1935 con el nombramiento de Gildardo Magaña como gobernador (Samaniego 1999b: 597; 1999a: 640). A partir de este momento, la iniciativa cardenista manifestada en el Plan de Recuperación acerca de la relación entre Baja California y Estados Unidos expresaba que los cambios debían empezar con iniciativas en la economía y en la vida política, a fin de que la población aumentara, viviera conforme al ritmo económico y social del centro del país y contuviera las amenazas de una economía con más avanzada, refiriéndose a California (Plan de Recuperación, 1936, en Cárdenas, 1978a: 215).

Con lo anterior se pronosticaron los cambios en la entidad y el advenimiento de la entrega de las tierras y conflictos laborales. Aunque, de manera sucinta, la Ley de Población fomentó el fortalecimiento de poblados fronterizos a través del aumento de la población mexicana para contrarrestar a la extranjera. ${ }^{16}$

\section{4) Las líneas de acción}

Al amparo de la Reforma Agraria cardenista, las principales líneas de acción y su instrumentación incluyeron el reparto de tierras cuya propiedad estaba en manos de compañías extranjeras, la creación del Distrito de Riego y redistribución del agua para usos agrícolas -que de manera paulatina tomó bajo su control el Estado mexicano-, y el apoyo financiero estatal para el desarrollo de la producción agropecuaria, que fue canalizado en forma de crédito a través de los bancos de crédito

${ }^{16}$ Artículo 7, VI, VII, así como en el 1º, III, IV, Ley General de Población, 1936. 
agrícola. Además se incluyó a los repatriados y migrantes de otras partes del país como posibles colonos o trabajadores.

Como ya se mencionó, entre las medidas que afirmaron la presencia del cardenismo en Baja California estaba la prohibición de los juegos de azar, lo cual incluía el cierre del hotel y casino Agua Caliente y los casinos establecidos en la Avenida "A" de Tijuana. Esto creó problemas de índole laboral e inconformidades de los trabajadores desempleados en este poblado (Samaniego, 1999a: 640).

Por otro lado, la Comisión Mixta Intersecretarial, ${ }^{17}$ creada con el objetivo de conocer la situación en que se encontraba Baja California, llegó a la conclusión de que debía realizarse la construcción de carreteras que unieran los poblados del Territorio, además que se trasladaran las oficinas de Pesca de San Diego y San Pedro, California, a Ensenada. Así también se fomentaron las inversiones en el puerto de Ensenada para desarrollo pesquero (Samaniego, 1999a: 642).

Las líneas de acción mencionadas -el reparto de tierras, la prohibición de juegos de azar y la creación de infraestructura- permitieron identificar los factores que influyeron en la movilidad poblacional de Baja California durante los años treinta. Desde una perspectiva centralista, se trataba de ir "mexicanizando" a la entidad a pesar de las trabas económicas y políticas.

\section{5) Declaración de intención para alcanzar los objetivos propuestos}

Las declaraciones de intención, expresadas en los informes de gobierno y muy concretamente en el mensaje dirigido por el presidente Cárdenas en el Plan de Recuperación, enfatizaron la necesidad de fortalecer el poblamiento de los Territorios del país, entre ellos Baja California. Para lograr la consolidación del programa que Cárdenas tenía planeado para Baja California, primero se propuso "[...] enlazar los territorios con otras entidades federales", para lo cual había que utilizar "los medios legales que están a su alcance para arbitrarse los fondos

\footnotetext{
${ }^{17}$ AGN, Dirección General de Gobierno, serie 2.300(30)10, caja 157, exp. 18, en IIHUABC [16.35]; Riquelme (SMGE, IIH-UABC [2.31]).
} 
necesarios [...]" (Plan de Recuperación, 1936, en Cárdenas, 1978a: 216). Es decir, en primera instancia había que unir a Baja California con el interior del país mediante vías de comunicación.

En segundo lugar, Cárdenas planteó una "protección arancelaria para estímulo de la industria", así como una "política fiscal encaminada a obtener una justa productividad de la propiedad raíz", además de "regular la tenencia de la tierra" (Plan de Recuperación, 1936, en Cárdenas, 1978a: 216). Lo anterior engloba lo que Cárdenas desarrolló en el documento dirigiéndose a cada una de las secretarías para dar instrucciones acerca de lo que se necesitaba realizar para la "reconstrucción integral" de Baja California.

Dentro de algunas recomendaciones que se creyeron sobresalientes o más importantes se mencionan las siguientes: a la Secretaría de Gobernación, Cárdenas le recomendó que las propuestas de gobernador tuvieran el requisito de ser nativos o residentes con una antigüedad de cuando menos cinco años a la fecha de nombramiento. A la Secretaría de Hacienda y Crédito Público se le propuso que se ampliaran las franquicias de los perímetros libres y se suprimieran las tarifas arancelarias que pudieran gravar su ingreso al país, así como ampliar las operaciones de crédito y construcción de obras por parte de los bancos Nacional de Crédito Agrícola, Nacional de Crédito Ejidal, Hipotecario y de Obras Públicas y de Crédito Popular.

Además, Cárdenas solicitó a la Secretaría de Educación Pública un plan completo donde quedaran comprendidas las escuelas preparatorias y técnico-industriales, así como la fundación de escuelas rurales donde hiciera falta. A la Secretaría de Guerra y Marina le requirió la proyección de obras necesarias y de guarniciones, así como campos de aterrizaje y "la idea de cooperar en el desenvolvimiento económico y social de los territorios" (Plan de Recuperación, 1936, en Cárdenas, 1978a: 218). A la Secretaría de Agricultura y Fomento le demandó el aumento de las comisiones para el estudio de los problemas de irrigación, así como un "sistema factible de colonización". A la Secretaría de Comunicaciones y Obras Públicas se le instó a gestionar la construcción de las carreteras de Tijuana, Tecate y Mexicali, de Ensenada, Maneadero y San Felipe, además de una carretera peninsular, que uniera los Territorios Norte y Sur, y una línea ferroviaria "que par- 
tiendo de Fuentes Brotantes, territorio norte de la Baja California, toque Punta Peñasco, para terminar en Santa Ana, Sonora" (Plan de Recuperación, 1936, en Cárdenas, 1978a: 219).

Por otro lado, al Departamento Forestal y de Caza y Pesca le pidió que realizara las disposiciones necesarias para que "a la mayor brevedad, se concentre en Ensenada todo el personal de caza y pesca que reside en San Diego y San Pedro; en la inteligencia de que proveerá a la oficina central de Ensenada, de los elementos indispensables para el servicio que le corresponde". Además de que dos guardacostas deberían quedar uno en Ensenada y otro en el golfo donde le señalara la Secretaría de Guerra y Marina. Por último, al Departamento de Salubridad Pública le solicitó un programa de "higienización de los territorios" para controlar las enfermedades endémicas, sobre todo el paludismo y la tuberculosis (Plan de Recuperación, 1936, en Cárdenas, 1978a: 220-221).

\section{6) La instrumentación}

Por último, las acciones concretas se tradujeron en el reparto de las tierras en la entidad, como una medida que Cárdenas aplicó al país en general. En Mexicali se entregó entre 1935 y 1940 la cantidad de casi 400 mil hectáreas y en Ensenada alrededor de 240 mil. ${ }^{18}$ En Mexicali, para 1937 fue entregada la tercera parte del territorio de la Colorado River Land Company a los productores ejidales. Entre 1937 y 1945 ya se había repartido el $85 \%$. En este periodo se formaron alrededor de 64 ejidos con 122285 has y 36 colonias con 65560 has. La cantidad de hectáreas repartidas variaba en tanto se tratara de ejidatarios o de colonos. Es decir, a los ejidatarios se les entregaban 20 has de riego como mínimo a cada uno y a los colonos les fueron otorgadas extensiones de entre 40 y 100 hectáreas.

En cuanto a los núcleos ejidales, les fueron repartidas tierras a partir de 1937, ya que anteriormente no existía el ejido como unidad de

\footnotetext{
${ }^{18}$ Los datos para Mexicali fueron tomados de Anguiano (1995: 113). En cuanto a los de Ensenada se consultó a Samaniego (1999a: 662-678). Estas 240 mil hectáreas es la suma de las tierras repartidas que Samaniego menciona por separado en la descripción de cada ejido.
} 
producción. En este año, en Mexicali fueron beneficiados alrededor de 4382 ejidatarios con 97121 has de tierra. Por su parte, en las colonias agrícolas se otorgaron en 193521576 has a 1198 colonos; en 1936 se repartieron 36152 has a 1398 colonos; y en 1937 se alcanzó la cantidad de 57152 has entregadas a 1400 colonos. Esto significa que el número total de beneficiados por entrega de tierras en el periodo de Lázaro Cárdenas fue de 8378 entre colonos y ejidatarios, a los cuales se les repartió un total de 73205 has (Anguiano, 1995: 99-101 y 112-113).

En la década de los años cuarenta, en Mexicali se repartieron alrededor de 442481 has a alrededor de 14,980 beneficiados, lo cual casi dobla el número de personas favorecidas aunque el número de hectáreas fue seis veces más grande. Se debe tomar en cuenta que los pequeños productores privados fueron ganando terreno en cuanto a contar con el beneficio del reparto de tierras (Anguiano, 1995: 113).

En Ensenada, por su parte, fueron repartidas alrededor de 239890 hectáreas a aproximadamente 735 personas con derecho a dotación en 11 ejidos. ${ }^{19}$ Por otro lado, no se puede determinar el número de repatriados que arribaron a Baja California, así como tampoco se puede distinguir en cuanto a las tierras entregadas a personas que vivían en la entidad, a los repatriados y a los migrantes recientes que emergieron de otras entidades. Sin embargo, el hecho de que se repartieran tierras que estaban en posesión de compañías extranjeras, tanto en Ensenada como en Mexicali, constituye un fenómeno de atracción migratoria, lo cual es un determinante dentro de la política cardenista en cuanto a la forma que se tuvo a bien utilizar para "poblar" y "mexicanizar" el territorio. Además, si se considera hipotéticamente tan sólo la cantidad de 8378 beneficiarios de tierras de Mexicali en relación al total de habitantes de Baja California -el cual pasó de 48327 a 78907 habitantes entre 1930 y 1940-, casi una tercera parte de los 30580 habitantes que aumentó se debe a la distribución o inmigración por reparto de tierras. Esto reafirma la hipótesis de la influencia de la Ley de Población en el poblamiento bajacaliforniano.

\footnotetext{
${ }^{19}$ Samaniego (1999a: 662-678) menciona que el censo de la Comisión Agraria Mixta reportó la cantidad de 1452 personas, 319 jefes de familia y 735 capacitados para recibir tierras.
} 
Acerca de los repatriados, se puede establecer la hipótesis de que éstos constituyeron un contingente de personas que hicieron crecer los poblados de Tijuana, Ensenada y Mexicali, a pesar de que su número no ha sido determinado. ${ }^{20}$ Estos repatriados fueron favorecidos en las administraciones de gobernadores provenientes del centro del país, como en la de Carlos Trejo y Lerdo de Tejada, de quien se dice que aprovechó cualquier irregularidad de la tenencia de la tierra en el Valle de Mexicali para formar colonias agrícolas y por consiguiente entregar tierras a repatriados. ${ }^{21}$ Ya en plena crisis económica, éstos constituyeron un problema de desempleo, aún así, se toma con reservas la afirmación de Jorge Bustamante (1988: 22), quien señala que llegaban contingentes como de cien familias a Tijuana para establecerse en la colonia Libertad. Santiago también incluye a las colonias Morelos y Castillo (Conferencia, 9 de mayo del 2000). Se concluye que hacen falta trabajos de corte cualitativo que puedan dar luz acerca del establecimiento de los repatriados.

Recapitulando, el reparto agrario fue una línea de acción de la política cardenista que, junto con el proceso de repatriación y migración, formaba parte de una política cuyo objetivo era aumentar el número de población en Baja California. Todo esto indica que las personas beneficiadas por el reparto fueron atraídas por el hecho de poblar la entidad, pero además se les dotó con las herramientas para lograr un arraigo, una forma de vida que expresaba lo que para Cárdenas significó "mexicanizar" a la entidad.

En el caso de la Ley de Población expedida en 1936, concretamente para Baja California, se establece que los seis puntos señalados por

\footnotetext{
${ }^{20}$ Bibiana Santiago Guerrero (Conferencia, 9 de mayo del 2000) sostiene la hipótesis de que los repatriados fueron muy importantes en el crecimiento de algunas colonias, en especial de las colonias Libertad, Morelos y Castillo. Con anterioridad, Samaniego (1989:130) menciona que el empobrecimiento que generó la crisis económica en Tijuana favoreció la formación de la colonia Libertad, la colonia Morelos y un barrio que se encontraba en la zona de Puerta Blanca al noroeste del poblado.

${ }^{21}$ Samaniego (1999b: 620) menciona que en el caso de la colonia Carlos Pacheco en Ensenada es arriesgado hablar del número de repatriados porque éstos se dedicaban a varias actividades, pero explica el caso de algunas afectaciones como la de la señora Bertha Marsh, parte de cuyas tierras fueron tomadas para repatriados.
} 
Miró (1975) tienen relación directa con ésta; además, es el antecedente que marca una coyuntura histórica en cuanto a considerar a la población objetivo de una política. En 1936, un año antes de que se efectuara la Reforma Agraria en Baja California, el gobierno federal tenía claro que el objetivo principal era "poblar" esta entidad. Basándonos en los puntos principales que establece Miró podemos llegar a la conclusión de que, efectivamente, existió una política de población en Baja California durante el periodo administrativo federal cardenista.

Al reunir los parámetros demográficos que se intentaban implantar en México después de un movimiento armado, una crisis económica internacional y un sistema político que se necesitaba fortalecer, la Ley General de Población de 1936 nutrió de elementos que hicieron viable la inmigración mexicana hacia Baja California, la cual se empezó a evidenciar en los periodos administrativos subsecuentes al del cardenismo.

\section{El periodo de 1930-1940 y su posible comportamiento}

Se ha observado hasta este momento que Baja California tuvo sus más altos rangos de crecimiento poblacional en la primera mitad del siglo pasado. No obstante, dicho crecimiento todavía se puede fraccionar aún más en dos oleadas de crecimiento: la que inicia desde antes de 1900 hasta la registrada en el censo de 1921, y la que va de esa fecha hasta 1950. El punto medio entre estas dos oleadas o disparos de crecimiento está enmarcado por un descenso que no significa una depreciación o baja, sino un crecimiento más lento en comparación con los anteriores. Se puede hipotetizar, por lo tanto, que factores externos influyeron para que la migración no tuviera la misma intensidad que la registrada en la década 1910-1921, o al revés, que el crecimiento de la década 1930-1940 se vio interrumpido por altas oleadas migratorias que aumentaron la velocidad del crecimiento. Ahora bien, considerando el movimiento armado de la Revolución, así como las políticas de puertas abiertas implementadas por Esteban Cantú en el periodo 1915-1920 y la crisis económica de 1929, y dejando de lado la inmigración a la entidad debido a la apertura del ferrocarril, el reparto de tierras ocurrido en 1936 y la ley poblacionista, ¿cuál hubiera sido el comportamiento de la población? 
A partir de esta pregunta, y en aras de comprobar si la política de población cardenista influyó en el poblamiento de Baja California, se realizó una estimación de la población desde 1895 hasta 1930, para incluir una alta inmigración de 1910 a 1921 y sumar la cantidad de habitantes de 1930. Esto permite tener una perspectiva de la cantidad de habitantes que hubiera habido ante una inercia demográfica que incluyera alta fecundidad y alta mortalidad pero una migración constante. Los datos se estimaron hasta el año 2000, sin embargo, en la figura 3 se observa hasta 1950 para tener un mayor detalle del periodo estudiado. No obstante, la cantidad a la que se hubiera llegado hacia el año 2000 con dicho comportamiento sería de 1445 046, habitantes, con una diferencia de los datos observados de 1078059 debido a que en este año fueron censados 2523105 habitantes.

La estimación se realizó a partir de la función logística ${ }^{22}$ y al compararse los datos del crecimiento con los datos observados y los estimados puede llegarse a la siguiente aseveración: el crecimiento de la población de Baja California hubiera sido menos acelerado que el crecimiento observado de haber continuado la tendencia hasta 1930. Como se observa en el cuadro 2, entre 1900 y 1921 las diferencias entre los datos observados y los estimados son negativas, lo que quiere decir que las cantidades de habitantes de los datos estimados fueron mayores a las observados, encontrándose la mayor diferencia en 1910 con -3 827 habitantes. Es decir, que de haber seguido la tendencia de 1895 y 1900, la población debió alcanzar los 13668 habitantes en 1910, y sin embargo, sólo llegó a tener 9841.

${ }^{22}$ La fórmula es $Y(t)=\frac{K}{1+e^{f(t)}}$ donde $Y(t)$ es la población en el momento, $t$ y $K$ es el límite máximo de variación. Se eligió la cantidad de 5,000,000 como el valor último de la asíntota superior. Por su parte, $f(t)$ es igual a una función lineal donde el parámetro es el tiempo, por lo que se convierte en la siguiente ecuación: $Y(t)=\frac{K}{1+e^{\alpha+\beta t}}$. Con la fórmula anterior se encontraron los valores de alfa y beta conforme la ecuación lineal de los mínimos cuadrados. De ello resultó la estimación con base en las cantidades de la población censada en Baja California desde 1895 hasta 1930, como se puede observar en la figura 3. La diferencia entre las funciones logística y exponencial es que la logística supone un límite o techo que forma una asíntota. 
Figura 3.

Estimación de la población de Baja California 1895-1950

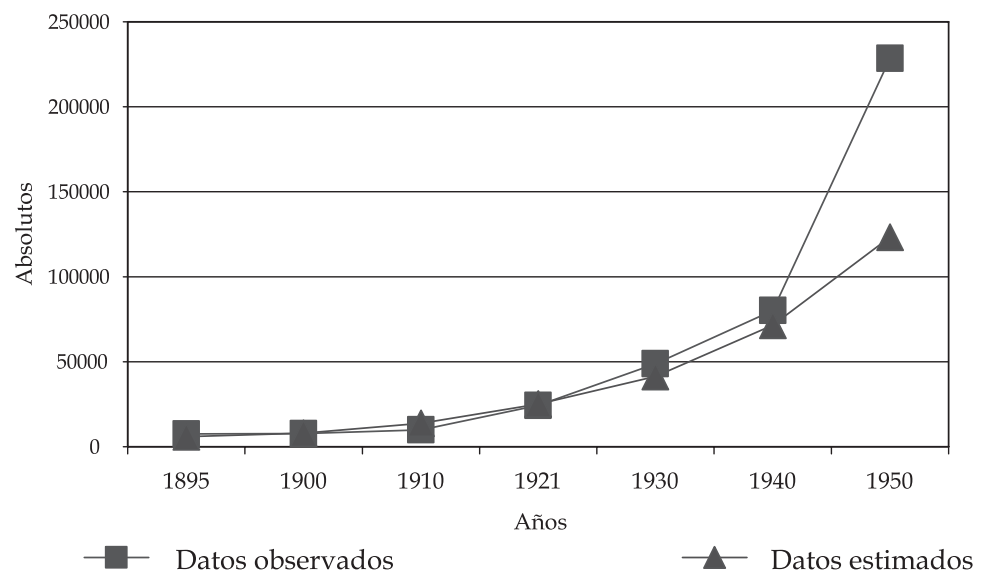

Fuente: Cuadro 2.

Al seguir estas cifras podemos verificar que a pesar de la última cantidad mencionada, en la siguiente década (1921) se pudo contemplar el mayor equilibrio con sólo 790 habitantes de diferencia, pero a partir de este momento se abriría cada vez más la brecha entre los datos intercensales. Es decir, que aunque originalmente haya existido menos población en 1910 con respecto a la tendencia de las décadas anteriores, para 1921 se recuperó casi al mismo nivel que los datos estimados, con 24333 habitantes que registró el censo y 25123 habitantes estimados.

Ya para 1930 es evidente que de haber seguido una tendencia similar desde principios del siglo, la población sólo debió llegar a los 41283 habitantes; no obstante, aumentó otras 7565 personas a pesar de haber tenido una tasa más baja de crecimiento que la década anterior. Entonces a partir de esta década la población estimada es menor que la observada: en 1940 se censaron a 80171 personas, pero los datos de la estimación nos dicen que debió haber 8664 habitantes menos. 
Cuadro 2.

Población de Baja California, 1895-1950

\begin{tabular}{lrrr}
\hline Años & Datos observados & Datos estimados & Diferencia \\
\hline 1895 & 7452 & 5950 & 1502 \\
1900 & 7592 & 7852 & -260 \\
1910 & 9841 & 13668 & -3827 \\
1921 & 24333 & 25123 & -790 \\
1930 & 48848 & 41283 & 7565 \\
1940 & 80171 & 71507 & 8664 \\
1950 & 228508 & 123309 & 105199 \\
\hline
\end{tabular}

Fuente: Cálculos propios a partir de los Censos de Población y Vivienda, 18951950.

El contraste más fuerte puede leerse en 1950, donde de haber seguido la tendencia del tiempo anterior sólo se hubiera alcanzado la cifra de 123309 habitantes; por el contrario, se llegó a la suma de 228 508. Esto significa que hipotéticamente, en vez de duplicarse la población entre 1940 y 1950, casi se triplica en la realidad.

La diferencia entre los datos de 1940 a 1950 hace alusión a la influencia de las políticas poblacionistas implementadas y estipuladas a partir de la Ley General de Población de 1936, ya que una política integradora permitió el reforzamiento de lazos económicos y sociales mediante el reparto agrario, los financiamientos, el impulso a la educación y la colonización con elementos nacionales, todo lo cual logró arraigar y hacer crecer a la población que ya existía y a la que inmigró.

Como se mencionó en párrafos anteriores, la tendencia demográfica real fue mucho más alta que la virtual, por lo que podemos ver el grado de influencia de la política de población cardenista en el poblamiento bajacaliforniano, sobre todo a través de la política de reparto agrario. En este periodo, de 1936 a 1940, se repartieron tierras a más de ocho mil beneficiarios tan sólo en Mexicali. Por añadidura, los saltos cuantitativos registrados hicieron que la entidad alcanzara el número uno en cuanto al nivel de las tasas de crecimiento a nivel nacional durante el siglo. 


\section{Conclusión}

Los problemas relacionados al tamaño de la población, la densidad y el crecimiento se encuentran dentro del debate demográfico actual. Durante el cardenismo esta problemática adquirió tal relevancia que se expidió la primera Ley General de Población. Determinar si esta ley y la política que la generó incidieron o no en la sociedad, reitera la vigencia del estudio de los fenómenos demográficos dentro de los procesos históricos. Aquí concluimos que pese a que se ha argumentado que las políticas del gobierno central no influyeron en el poblamiento de Baja California (Canales, 1995:5-23), hemos demostrado que sí influyeron.

Si el crecimiento de las primeras tres décadas del siglo xx hubiese seguido la tendencia demográfica, la población no hubiera aumentado en la proporción en que lo hizo. Sin embargo, el énfasis otorgado por el gobierno central después de los años treinta y el proceso de industrialización posterior a los años cuarenta motivaron el crecimiento vertiginoso que caracteriza a Baja California. Por tanto podemos concluir que tanto la política de población como la Reforma Agraria del periodo cardenista propiciaron un poblamiento en Baja California.

Se puede afirmar que la tasa de crecimiento del 5\% entre 1930-1940 fue una coyuntura histórico-demográfica del siglo xx. Antes de este periodo, el poblamiento se debió al proceso histórico que impulsó el nacimiento de las ciudades de Tijuana y Mexicali iniciado en el periodo del Porfiriato. Después de 1930, la población observada creció más de siete mil personas con respecto a la estimada en toda la entidad. ${ }^{23}$ Por todo lo anterior afirmamos que después de 1940 se empezaron a cosechar los logros de una política de población integradora cuya estrategia fue lograr el impulso económico de la entidad -reparto de tierras, financiamiento ejidal, apoyo al sistema educativo, escenario de preguerra, desarrollo del comercio en zona libre- y colonizar el territorio.

\footnotetext{
${ }^{23}$ Según los datos estimados para 1930 la población sólo debió llegar a los 41283 habitantes, pero alcanzó los 48848 habitantes, es decir, aumentó alrededor de 7565 personas. A partir de esta década la población estimada es menor que la observada: en 1940 se censaron a 80171 personas, pero los datos de la estimación nos dicen que debió haber cerca de 8664 habitantes menos.
} 


\section{Bibliografía}

Alanís Enciso, Fernando Saúl (2000), El gobierno de México y la repatriación de mexicanos de Estados Unidos (1934-1940), México, tesis de doctorado en Historia, Colmex.

Anguiano, Arturo (1984), El Estado y la política obrera del cardenismo, México, Era.

Anguiano, María Eugenia (1995), Agricultura y migración en el Valle de Mexicali, Tijuana, El Colef.

Bonifaz de Novelo, María Eugenia (1999), "El gobierno de Esteban Cantú, 1915-1920", en Marco Antonio Samaniego (coord.), Ensenada: nuevas aportaciones para su historia, Mexicali, UABC.

Bonifaz, Roselia (1999), "Conformación del Distrito Norte de Baja California, 1887-1911", en M. A. Samaniego (coord.), Ensenada: nuevas aportaciones para su historia, Mexicali, UABC.

Bustamante, Jorge A. (1988, 9 y 10 de julio), "Surgimiento de la colonia Libertad en la historia de México", memoria del Primer Simposium de Historia Documental de Tijuana, Tijuana, XII Ayuntamiento de Tijuana, CIH-UNAM/UABC.

Calvillo, Max (1994), Gobiernos civiles del Distrito Norte de la Baja California, 1920-1923, México, INEHRM, Secretaría de Gobernación. (2002), "Los gobernadores del Distrito Norte, 1920-1923", en Catalina Velázquez (coord.), Baja California. Un presente con historia, Mexicali, UABC.

Canales Cerón, Alejandro (1995), "El poblamiento de Baja California. 1848-1950", Frontera Norte, vol. 7, núm. 13, enero-junio, pp. 523.

Cárdenas, Lázaro (1978a), “Exposición del presidente de la República sobre la reconstrucción integral de los territorios de Baja California y Quintana Roo. México, D. F., 28 de septiembre de 1936" [Plan de Recuperación, 1936], en Palabras y documentos públicos de Lázaro Cárdenas, 1928-1970. Mensajes, discursos, declaraciones, entrevistas y otros documentos. 1928-1940, México, Siglo XXI. (1978b), “Mensaje de Lázaro Cárdenas al Congreso de la Unión al tomar posesión de la primera magistratura del país, México, 
D. F., 30 de noviembre de 1934" [Mensaje al Congreso, 1934], en Palabras y documentos públicos de Lázaro Cárdenas, 1928-1970. Mensajes, discursos, declaraciones, entrevistas y otros documentos. 19281940, México, Siglo XXI.

Cruz González, Norma del Carmen (2004), Baja California en el contexto de la política de población durante el periodo cardenista, 1930-1940, Tijuana, tesis de maestría en Demografía, El Colef.

Dirección General de Estadística (1913), Tercer censo de población de los Estados Unidos Mexicanos, Distrito Norte de la Baja California Secretaría de Hacienda, verificado el 27 de octubre de 1910.

Gómez Estrada, José Alfredo (2002), Gobierno y casinos, el origen de la riqueza de Abelardo L. Rodríguez, México, UABC, Instituto Mora, col. Historia urbana y regional.

González Félix, Marisela (2002), “Empresarios y gobierno en el Distrito Norte, 1902-1920", en C. Velázquez (coord.), Baja California. Un presente con historia, Mexicali, UABC.

INEGI (1997), División territorial del estado de Baja California de 1810 a 1995, Aguascalientes.

Lemoine Villicaña, Ernesto (1959), “Evolución demográfica de la Baja California”, Historia Mexicana, núm. 34, octubre-diciembre, pp. 249-268.

Ley General de Población (1936), México, Cámara Nacional de Comercio e Industria de la Ciudad de México.

Loyo, Gilberto (1935), La política demográfica de México, México, Instituto de Estudios Sociales, Políticos y Económicos del PNR.

Martínez García, Gerónimo (1987), Evolución de la política de población en México, México, Conapo.

Miró, Carmen (1975), Política de población: ¿qué?, ¿por qué, ¿para qué?, ¿cómo?, Celade, serie A, núm. 110, octubre.

Piñera Ramírez, David (1991), Ocupación y uso del suelo en Baja California. De los grupos aborígenes a la urbanización dependiente, México, UNAM, CIH-UABC.

Riquelme, Julio (1933), “El Territorio Norte de la Baja California y sus principales problemas", SMGE, Boletín de la Sociedad Mexicana de Geografía y Estadística, México, Editorial Cultura, pp. 187-205, en SMGE, IIH-UABC [2.31]. 
Rodríguez, Abelardo L. (1993), Memoria administrativa del gobierno del Distrito Norte de la Baja California, 1924-1927, Mexicali, SEP-UABC, 2da. ed., col. Baja California: Nuestra Historia.

Samaniego, Marco Antonio (1999a), "El cardenismo en Ensenada: el establecimiento del Estado corporativo", en M. A. Samaniego (coord.), Ensenada: nuevas aportaciones para su historia, Mexicali, UABC.

(1999b), "La emergencia de la crisis económica y los nuevos actores políticos, 1930-1935. Los años del maximato", en M. A. Samaniego (coord.), Ensenada: nuevas aportaciones para su historia, Mexicali, UABC.

(1999c), "La formación de la burguesía revolucionaria: el gobierno de Abelardo L. Rodríguez", en M. A. Samaniego (coord.), Ensenada: nuevas aportaciones para su historia, Mexicali, UABC. (1999d), "Los gobiernos civiles: una demanda de los comerciantes ensenadenses, 1920-1923", en M. A. Samaniego (coord.), Ensenada: nuevas aportaciones para su historia, Mexicali, UABC. (1998), Los gobiernos civiles en Baja California, 1920-1923. Un estudio sobre la relación entre los poderes local y federal, Mexicali, UABC. (1989), "Surgimiento, luchas e institucionalización del movimiento obrero en Tijuana. 1920-1940", en Jesús Ortiz Figueroa y David Piñera Ramírez (coords.), Historia de Tijuana, 1889-1989. Edición conmemorativa del centenario de su fundación, Tijuana, CIH, UNAM, UABC.

Santiago Guerrero, Bibiana (2000, 9 de mayo), "Colonias antiguas de Tijuana: fundación y migración de familias, 29 de julio de 1929”, Conferencia presentada en el ciclo Historia de Baja California: una experiencia compartida, Tijuana, IIH-UABC, Sociedad de Historia de Tijuana.

Taylor, Lawrence (2002), "La creación del estado de Baja California", en C. Velázquez (coord.), Baja California. Un presente con historia, Mexicali, UABC.

Velázquez, Catalina (2001), Los inmigrantes chinos en Baja California, 1920-1937, Mexicali, UABC. 


\section{Otros documentos}

AGN, Dirección General de Gobierno, serie 2.300(30)10, caja 157, exp. 18, en IIH-UABC, [16.35].

AGN, fondo Lázaro Cárdenas, vol. 488, exp. 437.1/413.

Fecha de recepción: enero de 2007

Fecha de aprobación: junio de 2007 